\section{NEUTRAL NETWORKS OF GENOTYPES: EVOLUTION BEHIND THE CURTAIN}

\author{
Susanna C. Manrubia \\ Centro de Astrobiología, CSIC-INTA \\ Ctra. de Ajalvir km. 4 \\ 28850 Torrejón de Ardoz, Madrid, Spain
}

José A. Cuesta

Grupo Interdisciplinar de Sistemas Complejos (GISC)

Dept. de Matemáticas

Universidad Carlos III de Madrid,

Avda. de la Universidad 30, 28911, Leganés, Madrid, Spain

\begin{abstract}
RESUMEN: Nuestra compresión de los procesos evolutivos ha progresado mucho desde la publicación, hace 150 años, de "El Origen de las Especies" de Charles R. Darwin. En el siglo XX se han realizado grandes esfuerzos para unificar la replicación, la mutación y la selección en el marco de una teoría formal, capaz de llegar a predecir la dinámica y el destino final de poblaciones en evolución. Sin embargo, la vasta evidencia experimental acumulada a lo largo de las últimas décadas indica, sin lugar a dudas, que algunas de las hipótesis de esos modelos clásicos necesitan una profunda revisión. La viabilidad de los organismos no depende de un único genotipo óptimo. El descubrimiento de enormes conjuntos de genotipos (o redes neutras) que dan lugar al mismo fenotipo -en última instancia, al mismo organismo- revela que, con una gran probabilidad, se puede encontrar soluciones funcionales muy diferentes, acceder a ellas y fijarlas en una población, mediante una exploración "a coste cero" del espacio genómico. Esta "evolución en la trastienda" podría ser la respuesta a algunos de los enigmas evolutivos a los que se enfrenta la teoría evolutiva, tales como los rápidos procesos de especiación que se observan en el registro fósil precedidos de largos periodos de estasis.
\end{abstract}

PALABRAS CLAVE: Red neutra; correspondencia fenotipo-genotipo; redundancia; adaptación; paisaje de fitness.

\section{INTRODUCTION}

The first name that comes to our minds when we hear the word "evolution" is Darwin. No doubt that Charles Robert Darwin's On the Origin of Species (1859), together with the sequels that he also published (The Descent of Man, The Expression of Emotions in Man and Animals...), form the cornerstone of our current understanding of the most fundamental process of life. Nevertheless, Darwin neither discovered evolution himself, nor was he the only one to propose the mechanism of natural selection to explain the

\section{REDES NEUTRAS DE GENOTIPOS: EVOLUCIÓN EN LA TRASTIENDA}
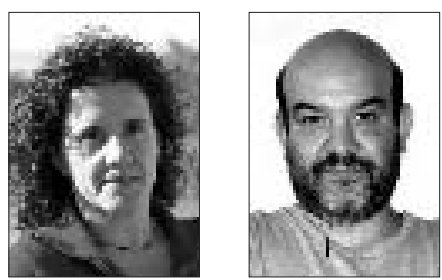

ABSTRACT: Our understanding of the evolutionary process has gone a long way since the publication, 150 years ago, of " $0 n$ the origin of species" by Charles R. Darwin. The XXth Century witnessed great efforts to embrace replication, mutation, and selection within the framework of a formal theory, able eventually to predict the dynamics and fate of evolving populations. However, a large body of empirical evidence collected over the last decades strongly suggests that some of the assumptions of those classical models necessitate a deep revision. The viability of organisms is not dependent on a unique and optimal genotype. The discovery of huge sets of genotypes (or neutral networks) yielding the same phenotype -in the last term the same organism-, reveals that, most likely, very different functional solutions can be found, accessed and fixed in a population through low-cost exploration of the space of genomes. The "evolution behind the curtain' may be the answer to some of the current puzzles that evolutionary theory faces, like the fast speciation process that is observed in the fossil record after very long stasis periods.

KEY WORDS: Neutral network; genotype-phenotype map; redundancy; adaptation; fitness landscape.

evolution of species. At Darwin's time, the fact that species evolved was common knowledge'. On the other hand, Alfred Russel Wallace published, simultaneously with Darwin, a theory of evolution based on what we currently know as natural selection, the same key idea put forward in "The Origin". Then, why is Darwin's work so fundamental for the current theory of evolution? To understand the depth of his contribution, one must read "The Origin" -just an abstract, in his words, of the work he intended to publish two or three years later (Darwin, 1859). He deserves the credit for this theory because of both the overwhelming 


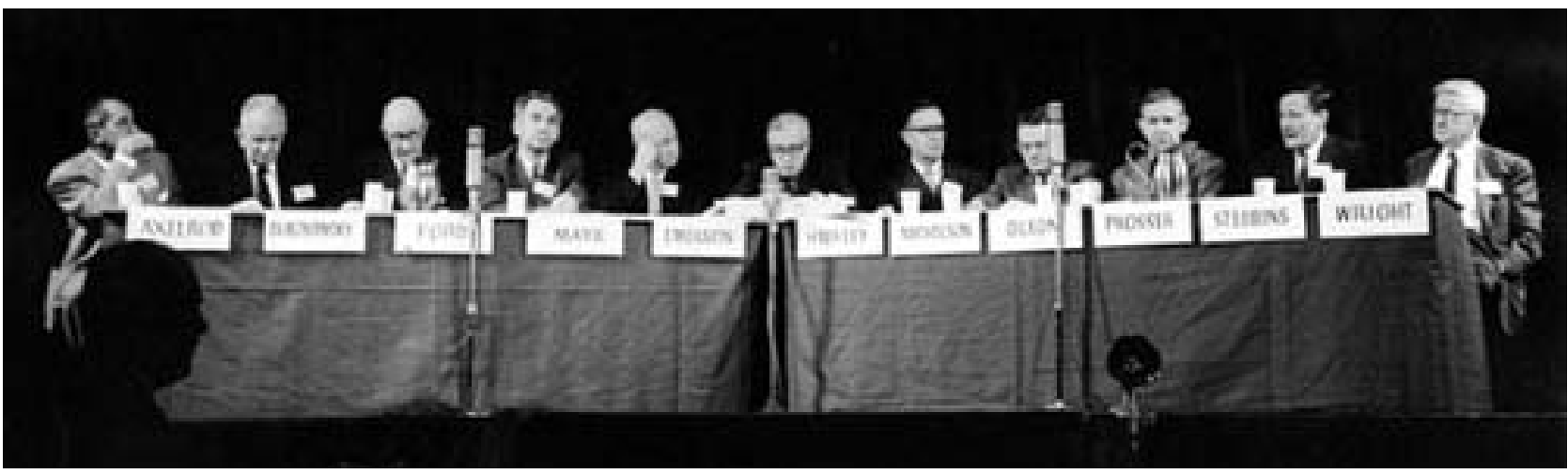

Figura 1. "The Evolution of Life", November 25, 1959. Darwin Centennial Celebration. From left to right: Daniel l. Axelrod, Theodosius Dobzhansky, Edmund B. Ford, Ernst Mayr, Alfred E. Emerson, Julian Huxley, Alexander J. Nicholson, Everett C. Olson, Clifford Ladd Prosser, George Ledyard Stebbins, and Sewall Wright.

accumulation of empirical data he presented and the clear explanations that his theory offered to many different -and at the time independent- observations: geographical diversity, artificial selection, coevolution of plants and insects, appearance of complex organs, instincts in man and animals. He gave a unified view of the complexity of life by means of a unique universal mechanism. Evolution by natural selection was endowed with a creative power far beyond what Darwin's predecessors, or even Wallace, had ever proposed (Gould, 2002). It is for this reason that there was a centennial celebration of the publication of this fundamental book (see Fig. 1) and the motive of last year's sesquicentennial celebration, in the internationally proclaimed Darwin year.

However, Darwin's theory was incomplete. All throughout "The Origin", Darwin bumps once and again into the same problem: the mechanism of inheritance. At Darwin's time the standard theory of inheritance in sexual organisms assumed that individuals roughly inherited an average of their parent's traits. Sir Francis Galton, one of Darwin's cousins, discovered the statistical phenomenon of regression towards the mean (Galton, 1886), according to which traits that deviate from the mean of a population revert to this value as they breed, in a few generations. This problem permeates his work and forces Darwin to resort to the isolation of populations in order to explain the appearance and maintenance of new species. It was unfortunate that Darwin was not aware of Mendel's discovery of the laws of inheritance, published almost simultaneously with "The
Origin" in an obscure Austrian journal (Mendel, 1866). Mendel laws would have solved many of Darwin's problems with the sustainment of diversity. In fact, the rediscovery of these laws by de Vries, Correns and von Tschermak in 1900 triggered a big deal of research, both theoretical and experimental, which led, by the middle of the XXth century, to the so-called "modern synthesis" (Huxley, 1942). This revision of Darwinism can be considered as a true scientific theory in the sense that it is based on population genetics, a quantitative formulation of the theory of evolution by natural selection under the mechanisms of genetic inheritance.

\section{The CURRent PARAdigM: POPULATION Genetics}

Population genetics is the creation of a group of statisticians among whom we find some of the big names of evolutionary theory: Fisher, Haldane, Wright, and later Kimura. The focus of this theory is to determine the fate of a population whose individuals reproduce with variability and struggle for survival in an environment which discriminates their traits, favoring ones over others. More precisely, population genetics assumes that populations live in a more or less exhausted environment which maintains the amount of individuals almost constant along generations. Individuals breed and their offspring inherit their traits according to genetic laws. Different traits have different survival probabilities, and the action of chance 
upon this biased set decides who dies with no descent and who survives and reproduces, and, among the latter, the number of offspring of each individual. New traits appear randomly, at a very low rate, through mutations of existing genes. From this point of view, evolution is, to a large extent, a result of the laws of probability, hence the intrinsic statistical nature of population genetics.

Population genetics stands as the first coherent and quantitative account of the theory of evolution, and still today provides the paradigm that scientists have in mind when thinking about evolution. The picture it draws is that of a population of entities which replicate at a rate that depends on selection pressures, i.e. a measure of how adapted are their traits to the environment. New traits appear at a very low rate through mutations. The process is random and therefore subject to historical contingency, which translates into another feature exhibited by the evolution of populations: genetic drift, or sampling noise. By this we mean the fact that even for a population with two traits replicating at the same rate -i.e. having the same fitness- and represented fifty-fifty, the ratio of the two traits will deviate from this equal ratio in the next generation. This process is especially important in small populations (for instance, in evolutionary bottlenecks), but it has always been considered a secondary effect in large populations. The paradigm yielded by population genetics has been very successful not only in Biology, but also in other disciplines which have borrowed it to explain related phenomena. Economics, Sociology, Linguistics, or Computer Science are a few examples of areas where evolution as a result of the combined effect of replication, selection, and mutation, has provided a new framework to understand collective dynamics or to devise applications to solve existing problems.

But population genetics also makes several implicit assumptions which have basically remained unquestioned and have thus become part of the standard thinking in this discipline. Explicit models in population genetics make use of a metaphor introduced by Wright: the fitness landscape. In brief, it is assumed that fitness is uniquely determined once the genotype and the environment are given, so if the environment remains unchanged, the fitness landscape becomes a mapping from genotype to the mean replication rate (interpreted as fitness) of the individuals carrying that genotype. Evolution is then the movement through that fitness landscape. But what does it move? This is the first implicit assumption of population genetics: evolution moves the population as a whole. The mutation rate is considered so low that a mutation causing a new allele gets fixed in the population before the next mutation occurs and introduces a new allele into play. Thus evolution is the movement of a homogeneous population throughout the fitness landscape. This implicit assumption is made explicit in several works aimed at describing the evolution of populations with the language of Statistical Mechanics (Barton and Coe, 2009; Sella and Hirsh, 2005). A second implicit assumption shows up when examining the basic models of population genetics. Fisher's Fujiyama landscape assumes, for instance, that there is an optimum genotype for which fitness is maximal, and any deviation from that genotype by point mutations only degrades that fitness, the more the larger the distance in configurational space (genotype distance is usually measured in terms of Hamming distance, i.e. the number of positions in which two sequences differ). Wright's rugged landscapes are thought of as hilly landscapes, with many mountains and valleys, tops being fitness maxima, again located at specific genotypes. Many theoretical models like Muller's ratchet (Muller, 1932) or Eigen's quasispecies (Eigen, 1971), which have been very influential in our current evolutionary thinking, strongly rely on this optimum genotype assumption of population genetics.

Gradualism is implicit in this evolutionary paradigm: evolutionary changes occur only through the gradual, slow accumulation of small changes caused by the very infrequent appearance of beneficial mutations (most mutations are just deleterious). Gradualism, an idea that Darwin took from Geology, is one of the strong arguments of "The Origin" in justifying why we are not able to see evolution at work. We cannot see it like we cannot see mountains erosion, and yet we know it exists. But gradualism is also one of the most controversial points of evolutionary theory because it conflicts with the fossil record, where species are observed to remain nearly unchanged for long stasis periods, only to be quickly (in geological terms) replaced by new species [something that has been termed punctuated equilibrium (Eldredge and Gould, 1972)].

Gradualism is only the tip of the iceberg. Perhaps it is so because a case can be made against it from the empirical evidence accumulated by more than a century of paleon- 
tological research and from the accumulated knowledge on non-parsimonious evolutionary mechanisms. Still, it is not the only difficulty that the paradigm of population genetics faces, nor is it the first one to show up. We will see immediately that the strongest body of evidence against many of the assumptions underlying population genetics comes from molecular biology. And it urgently calls for a change of paradigm. This does not mean that population genetics is wrong: on the contrary, the tools it provides are still valid. It is only the picture it draws, more based on somehow prejudicial assumptions and on misleading metaphors, that is essentially incorrect.

\section{The NeW PARAdigm: neUtral EVOLUTION}

In 1968 Kimura surprised the scientific community with the argument that most mutations in the genome of mammals have no effect on their phenotype (Kimura, 1968): in other words, most mutations are neutral, neither beneficial nor deleterious. The argument goes as follows. Comparative studies of some proteins indicate that in chains nearly 100 aminoacids long a substitution takes place, on average, every 28 million years. The typical length of a DNA chain in one of the two sets of mammal chromosomes is about 4 billion base pairs. Every 3 base pairs (codon) code for an aminoacid and, because of redundancy, only $80 \%$ base pair substitutions give rise to an aminoacid substitution in the corresponding protein. Therefore there are 16 million substitutions in the whole genome every 28 million years; in other words, approximately a substitution every 2 years! Kimura concluded that such an enormous mutational load can only be tolerated if the great majority of mutations are neutral.

Subsequent studies with different systems (we will see later the case of RNA molecules) support this conclusion. At least at the molecular level, neutrality seems to be the rule, rather than the exception, thus contradicting the homogeneity assumption of population genetics. One could argue that neutral mutations can simply be disregarded, so that we can just focus on those that do produce phenotypic change in the individual. This might be an appropriate description of what is going on if the effect of mutations on phenotype, and therefore on fitness, could be added up, as if genes were simple switches of different traits that can be turned on and off by mutations (an unfortunately widespread misconception of how genes work). But things are far less simple. It turns out that genes are involved in a complex regulatory network in which the proteins codified by some genes activate or inhibit the coding of other proteins (even themselves), so that the action of a single protein -hence of a gene- cannot be disentangled from the action of very many others. In fact, there is nearly no single trait in multicellular animals or plants which is not the consequence of the combined effect of many genes acting together in this complex way.

The phenotype is thus the effect of the genome as a whole, rather than "a linear combination" of traits. Now, the accumulation of neutral mutations motivates that apparently similar individuals of the same species bear genomes that may be very far apart from each other. In this situation a new mutation may induce a big phenotypic change in one of these individuals but not in others because the net effect is as if the genome as a whole had been modified in just one step (all previous mutations were silent). This effect challenges the standard picture of gradualism and makes a case for punctuated equilibrium. Not only that: the idea that there is an optimum genotype makes no sense under such a wide neutral wandering in the space of sequences, and this, as we will see, questions many commonly accepted models in population genetics.

\section{VARIABILITY AND REDUNDANCY}

Biology is extremely redundant, and it is so at all its levels of complexity. We have just mentioned the redundancy of the genetic code. Every codon codes for an aminoacid using an almost universal code (see Fig. 2). Setting aside three "stop" codons (which mark the end of the gene), this implies that 61 codons code for only 20 aminoacids. Thus most aminoacids are coded by two, four, or even six codons, so many base pair substitutions in the DNA do not alter the coded protein. Proteins, in their turn, fold in an almost rigid three-dimensional structure (the so-called tertiary structure). This folding is induced by the interaction between the sequence of aminoacids conforming their primary structure. But not all aminoacids play the same role in folding the protein: some of them are critical, in the sense that if they are replaced by others the conformation 
of the protein changes, but most are nearly irrelevant, in the sense that their replacement leaves the protein unchanged or nearly so. As the tertiary structure determines the protein function, it turns out that many aminoacid substitutions do not modify the structure, and thus have no biological effect. Proteins then enter complex regulatory or metabolic network in which they interact with other proteins regulating their coding or participating in metabolic pathways. But then again some of this proteins may be replaced by other similar proteins with no major change in the network function.

\begin{tabular}{|c|c|c|c|c|c|}
\multicolumn{1}{c|}{ base 1} & base 2 & \multicolumn{3}{c|}{ base 3} \\
\hline \multirow{4}{*}{ T } & T & C & A & G & \\
\hline \multirow{4}{*}{} & PHE & SER & TYR & CYS & T \\
\cline { 2 - 6 } & PHE & SER & TYR & CYS & C \\
\cline { 2 - 6 } & LEU & SER & stop & stop & A \\
\cline { 2 - 6 } & LEU & SER & stop & stop & G \\
\hline \multirow{4}{*}{ C } & LEU & PRO & HIS & ARG & T \\
\cline { 2 - 6 } & LEU & PRO & HIS & ARG & C \\
\cline { 2 - 6 } & LEU & PRO & GLN & ARG & A \\
\cline { 2 - 6 } & LEU & PRO & GLN & ARG & G \\
\hline \multirow{4}{*}{ A } & ILE & THR & ASN & SER & T \\
\cline { 2 - 6 } & ILE & THR & ASN & SER & C \\
\cline { 2 - 6 } & ILE & THR & LYS & ARG & A \\
\cline { 2 - 6 } & ILE & THR & LYS & ARG & G \\
\hline \multirow{4}{*}{ G } & VAL & ALA & ASP & GLY & T \\
\cline { 2 - 6 } & VAL & ALA & ASP & GLY & C \\
\cline { 2 - 6 } & VAL & ALA & ASP & GLU & A \\
\cline { 2 - 6 } & VAL & ALA & ASP & GLU & G \\
\hline
\end{tabular}

Figura 2. Genetic code. A, T, G, C stand for the four basis of DNA (Adenine, Thymine, Guanine, and Cytosine). Transcription is carried out by RNA chains, which are copies of one DNA strand with $T$ replaced by $U$ (Uracil). PHE, $L E U$, ILE, etc., are abbreviations of the 20 aminoacids (PHEnylalanine, LEUcine, IsoLEucine, etc.). Codons labelled "stop" signal the end of transcription.

This extraordinary redundancy of biological systems makes them very robust to change. This is the origin of neutrality. In order to understand how much room for neutrality is there in biological systems and grasp some of the effects induced by variability we will closely examine a relatively simple example to which a big deal of research has been devoted to in the last decades: RNA folding (Ancel and Fontana, 2000; Fontana, 2002; Schuster, 2006). An RNA molecule is a chain formed by a sequence of the four nucleotides G, C, A, and U. Although it can form double chains, as DNA does, RNA molecules are usually single stranded. Nucleotides in an RNA sequence tend to form pairs to minimize the free energy of the molecule. This so-called secondary structure of RNA molecules determines to a large extent their chemical functions, and as such has been often used as a crude representation of the phenotype.

An upper bound for the number $S(I)$ of sequences of length I compatible with a fixed secondary structure is $S(I) \propto I^{-3 / 2} b$ (Schuster et al., 1994), where $b$ is a constant that depends on geometric constraints imposed on the secondary structure (e.g. the minimum number of contiguous pairs in a stack). The calculation of $S(I)$ is done in a recursive manner, summing over all possible modifications of a structure when its length increases in one nucleotide. The resulting equations may be considered a generalization of Catalan and Motzkin numbers (Waterman and Smith, 1978). The values of $S(I)$ for moderate values of / are certainly huge: there are about $10^{28}$ sequences compatible with the structure of transfer RNA (which has length $I=76$ ), while the currently known smallest functional RNAs, of length $I \approx 14$ (Anderson and Mecozzi, 2005), could in principle be obtained from more than $10^{6}$ different sequences. Figure 3 portrays a computational example of sequences folding into the same secondary structure, of length $I=35$ in that case. Note that the similarity between sequences may be very low, even if they share their folded configuration: a random subsample of a population reveals that sequences differ on average in 10 to 15 nucleotides, while differences up to $100 \%$ are possible.

All this enormous variability that redundancy supports may have a measurable effect: the equilibrium configuration of either large populations or of populations evolving at a high enough mutation rate, is very heterogeneous. For the sake of illustration let us consider a population of size $N$ undergoing a mutation rate $\mu$ per generation and per individual. To simplify, let us also assume that all mutations are neutral. In this case, the time $t_{g}$ in number of generations required for a mutation to spread to all individuals (or to disappear) is proportional to the population size, $t_{g} \simeq 2 N$ (Ewens, 2004). Now, the number $M$ of mutants that appear in this characteristic time is $M \simeq t_{g} \mu N=2 N^{2} \mu$. 


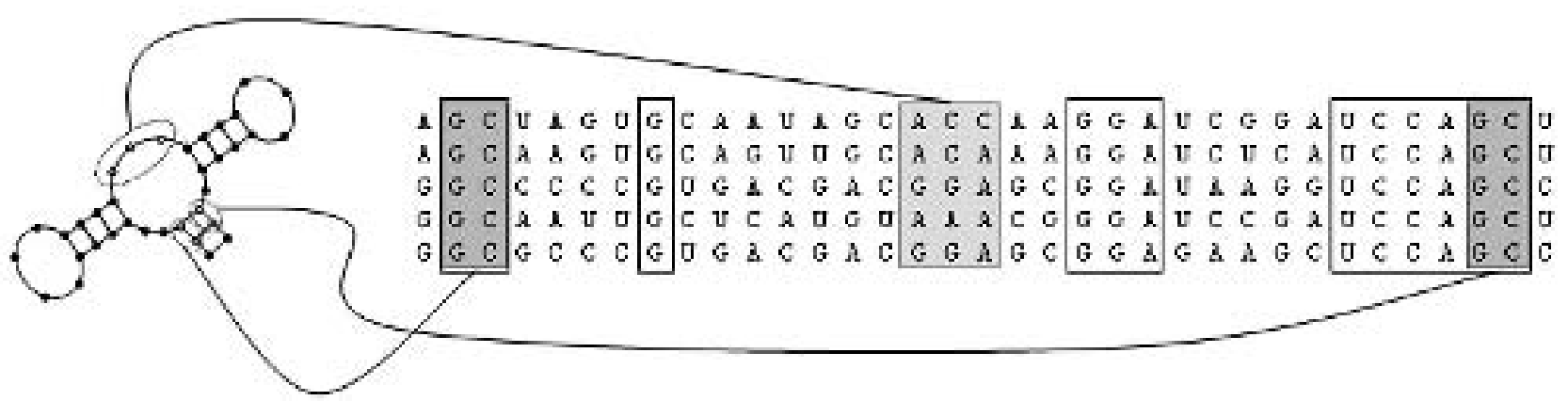

Figura 3. An example of an RNA secondary structure and a few of the sequences that fold into that state as their configuration of minimum energy. We highlight the only conserved regions in this example (nucleotides surrounded by solid-line boxes), which typically correspond to nucleotides forming pairs in the structure (a particular case shown in dark grey). Non-paired nucleotides form loops in the secondary structure, and are less conserved on average than stacks (e.g. the three positions forming the internal loop, indicated in light grey in the sequences).

The conclusion is straight: if $M \sim 1$ the population will be homogeneous most of the time, but if $M \gg 1$ mutants appear at a rate faster than that at which mutations are fixed in the whole population, so the statistical equilibrium will correspond to a heterogeneous population.

Heterogeneity is dynamically maintained not only in neutral characters, but also in features that affect fitness. There are abundant observations of suboptimal phenotypes that coexist with better adapted phenotypes. This is also a result of a high mutation rate that translates into nonzero transition probabilities between phenotypic classes. In other words, the existence of just one of the phenotypes generates all the others, which are mutually maintained at equilibrium. This type of organization is called quasispecies. It was first introduced in a theoretical setting to describe the organization of macromolecules at prebiotic times (Eigen, 1971), and the concept was subsequently applied to viruses (Domingo et al., 1978). Actually, RNA viruses yield abundant examples of heterogeneity, both in sequences and in function. The common situation is that each genotype is unique in the population, differing in at least one nucleotide from any other. But the isolation of those genotypes and the subsequent generation of clonal populations that descend from each of them reveals a high variability in phenotypic properties (replication time or virulence, for instance), such that the population is a heterogeneous ensemble in genotype and phenotype (Duarte et al., 1994).

\section{Distribution OF PHENOTYPES IN GeNOTYPE SPACE}

Genotype spaces are rather complex objects amenable to a deceptively simple description. So complex and so simple that thinking of them may easily lead to misleading images that misguide our intuition. Much of our difficulty in understanding the dynamics of evolving systems lies in these objects.

Consider a biological sequence of length $L$. Position $i$ of this sequence can adopt one out of $k$ variants, that we can think of as letters of an alphabet. Depending on the type of sequence this alphabet may be formed by the $k=4$ bases of which DNA or RNA are made of, by the $k=20$ aminoacids that build up proteins, or even by the different alleles of gene $i$ of a given chromosome. The description is similar in any of these instances but we shall focus e.g. on DNA to fix ideas. Every realization of a DNA sequence is a genotype, and represents a "point" in genotype space. There are $4^{L} L$-long different genotypes; if $L=100$, for instance -a rather short sequence, by the way, the size of the genotype space is $4^{100} \approx 10^{60}$, a huge set. Movement across this space proceeds through mutations. Mutations can be very complicated transformations of a genotype that can even modify its length, but again, to keep it simple we shall constraint ourselves to consider only point-like mutations, i.e. substitutions of the letter at a given position by another one of the alphabet. If we now make a graph whose nodes are all possible sequences and whose links join sequences 
(a)

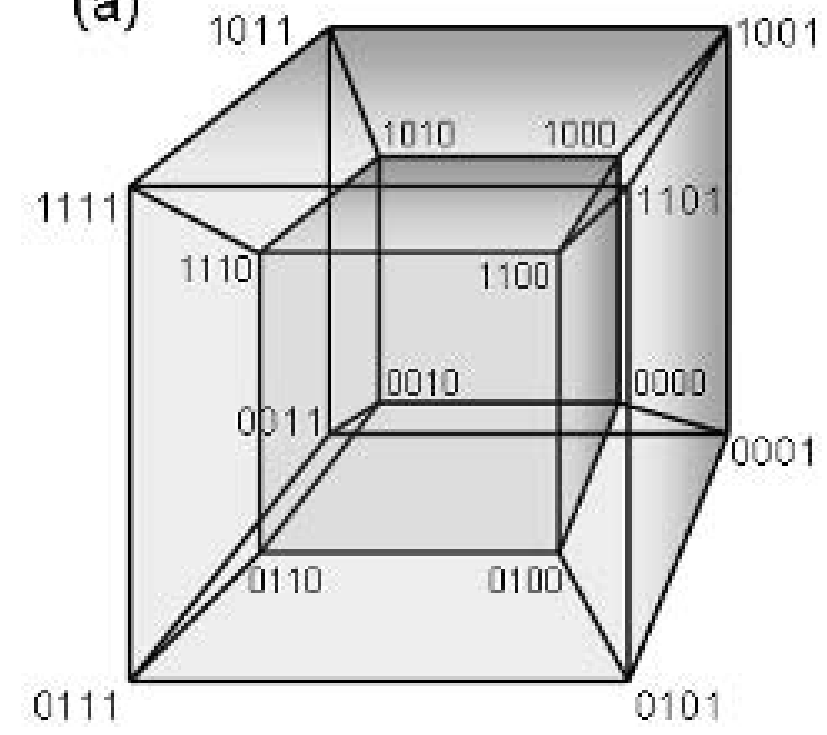

(b)

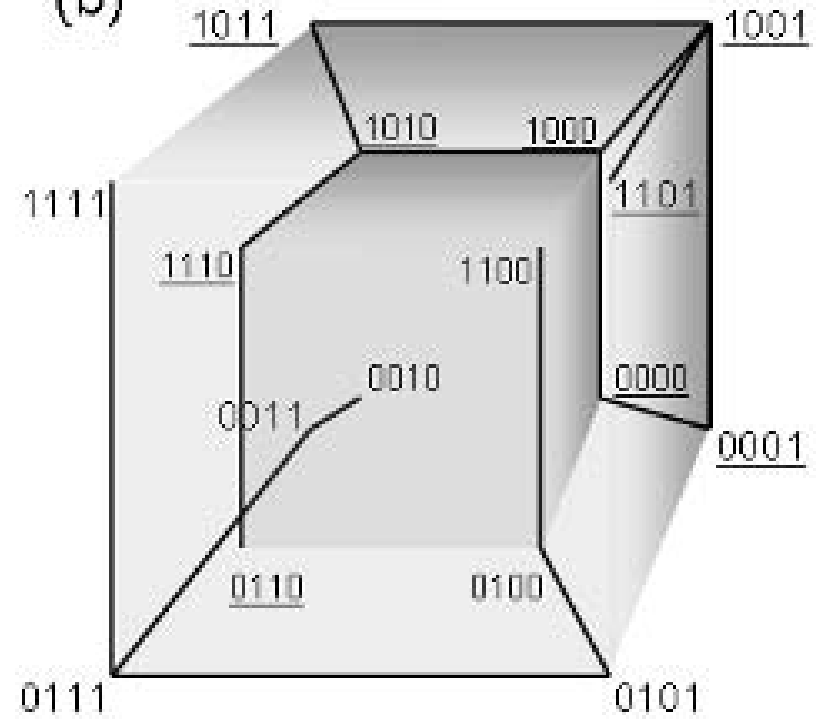

Figura 4. (a) Genotype space for a sequence of length $L=4$ and an alphabet of $k=2$ letters, $\{0,1\}$. (b) An example of how this space could split into two different neutral networks, each yielding a different phenotype. One of the networks contains the underlined nodes, the other network contains the remaining ones.

separated a by a point-like mutation, we have a topological description of a genotype space (Fig. 4 represents one of these spaces for $L=4$ for an alphabet with only two letters). Mutations move the sequence from a node of this graph to one of its $D=3 L$ neighbors which differ from it in just one position. In general, the genotype space is a regular lattice in an Euclidean space of dimension $D=(k-1) L$.

The huge size and high dimensionality of sequence spaces have non-trivial implications for the distribution of phenotypes in genotype space. Sequences with the same phenotype have therefore the same fitness, so a sequence can move across any connected component of the graph corresponding to one phenotype at no cost in fitness. Figure $4(b)$ yields a very simple example of sequences that can be accessed without changing the fitness of an individual. Note that a single mutation causes no changes if the mutated genome belongs to the same neutral network than its parental genome. However, in regions where two different networks are close, point mutation may generate a genome that belongs to a different network, such that major novelties in phenotype arise.
In order to better understand what these connected components look like let us consider a simple model in high dimensions -i.e. for genomes which are longer than those of Fig. 4. Let us assume that sequences are randomly and independently assigned to phenotypes, and let $p$ be the fraction of sequences corresponding to a given phenotype $\Phi$. Due to the complexity of the genotype space we can locally regard it as a tree (see Fig. 5). Given a node, each of its $D$ neighbors has $D-1$ new neighbors; each of these second neighbors of the first node will have, in its turn, $D-1$ new neighbors; and so on. Now, because nodes belong to $\Phi$ randomly and independently of each other, assuming that the first node belongs to $\Phi$, each second, third, etc., neighbor will also belong to $\Phi$ with probability $p$. If $(D-1) p>1$, on average every $\Phi$-node will have another $\Phi$-node among its neighbors, so the set of $\Phi$-nodes contains a connected cluster with a finite fraction of all the nodes of the graph. On the contrary, if $(D-1) p<1$ eventually the number of $\Phi$-nodes will drop to zero, and so the set of $\Phi$-nodes will be made of "small" disconnected clusters. Notice that the critical fraction of nodes is $p_{\mathrm{c}} \approx D^{-1}$, a very small number in high-dimensional spaces, so what we have just described is the typical situation. 


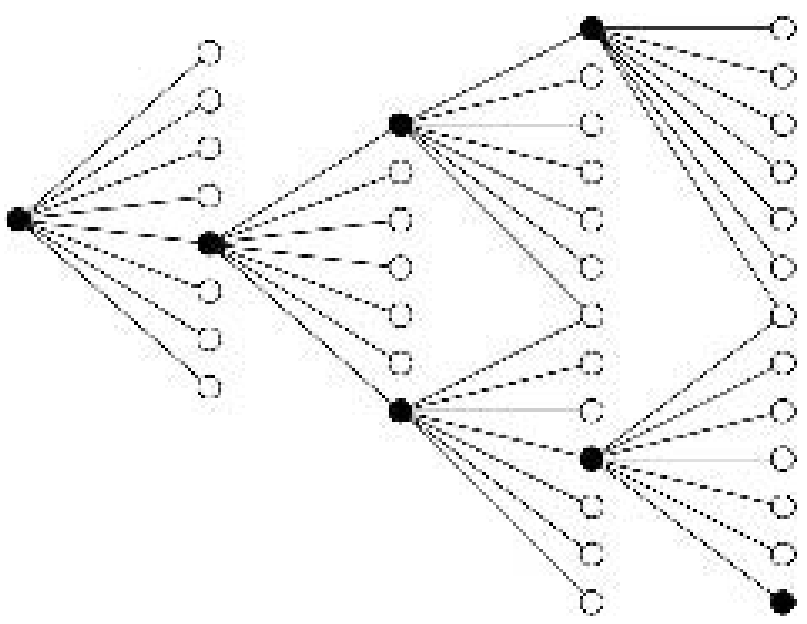

Figura 5. Model of the Russian roulette. Black nodes belong to the same phenotype $\Phi$, whereas white nodes correspond to different phenotypes (hence to different fitness values, in principle). If the fraction $p$ of black nodes times the number $D$ of links to nearest neighbors is above 1, the cluster of black nodes extends all over the network.

The picture this provides is very different from that of the standard fitness landscapes employed in population genetics. Here genotype spaces should be thought of as a patchwork of different phenotypes, each patch containing a finite fraction of the total set of nodes, all of which have the same fitness. Patches are intertwined in very irregular ways.

Again, RNA folding can give us a quantitative picture of how a neutral network of genotypes should look like, and how different networks are interrelated. Suppose that one can construct the complete mapping of RNA sequences of a given length to the secondary structures they fold into. The genome space would be partitioned into a large number of neutral networks, as sketched in Fig. 6 . The size of neutral networks varies broadly around an average of $(4 / b)^{1 / \beta / 2}$ sequences per network. For example, in the case of sequences of length $I=35$, there are around $10^{3}$ structures (called common structures) which are a thousand-fold more frequently obtained from the folding of a randomly chosen sequence than a background of millions of other structures that are yielded by few selected sequences (Stich et al., 2008). Interestingly, the functional structures found in Nature, though arising from a long and demanding selection process through geological time, all belong to the set of common structures. The network of genotypes corresponding to common structures traverses the whole space of genomes. In practice, thus, a population can contain a huge number of different genotypes with identical selective value. Populations can spread in the space of genomes without seeing their fitness affected. One important implication of the above is accessibility: almost any other possible secondary structure can be accessed with one or few changes in the sequence, since networks belonging to different folds have to be necessarily close to one or another of the common structures. Systematic measures with RNA structures indicate that any common structure lies at most $R$ nucleotides apart, with $R \simeq 0.21$, of any other randomly chosen common structure (Grüner et al., 1996).

Evidence for the spread of neutral networks throughout the sequence space, and for the existence of sequences performing different chemical functions (thus having different phenotypes) that lie just a few nucleotides apart, comes not only from RNA, but also from empirical results with aptamers and ribozymes. In a revealing experiment, Schultes and Bartel (2000) discovered close contacts between the neutral networks representing a class-III self-ligating ribozyme and that of hepatitis- $\delta$ virus self-cleaving ribozyme. The experiment began with the two original RNA sequences of the corresponding functional molecules, which had no more than the $25 \%$ similarity expected by chance. After about 40 moves in genome space, they located an intersection between the two neutral networks where two sequences just two nucleotides apart could perform the original functions without a major loss in fitness. This observation has been repeated in several other systems [see Schuster (2006) for a review].

An illustration of the relationship between genomes, neutral network spreading and phenotypes is represented in Fig. 6. Even in this two-dimensional representation it is clear how moving on a neutral network (thus conserving fitness) permits to access different phenotypes in a single mutational move. This property might underlie punctuated equilibrium, explaining the sudden changes in phenotypes observed after long periods of stasis (Fontana and Schuster, 1998). The movement of the population on the neutral network, though having effects at the genomic level, does 

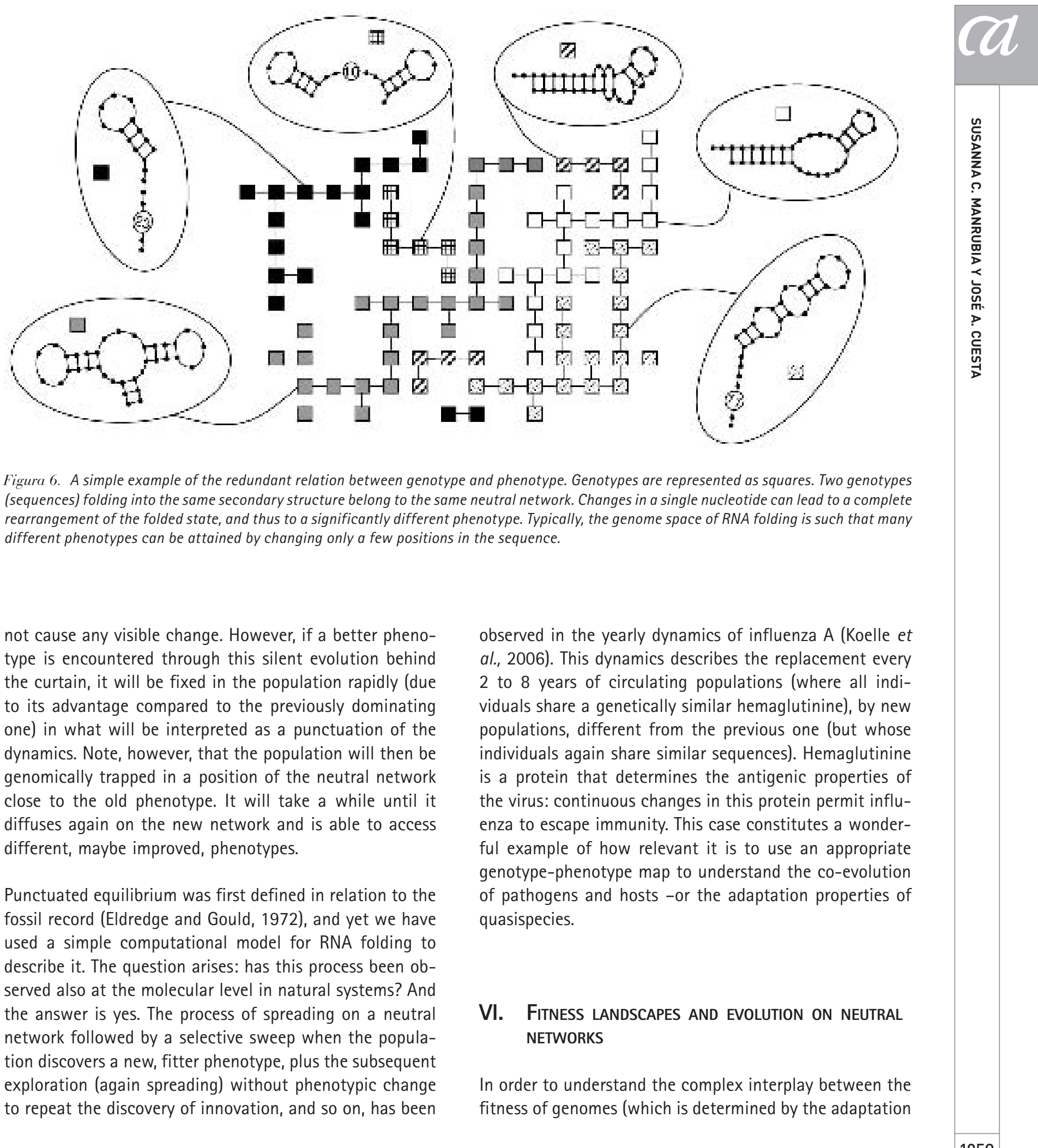

Figura 6. A simple example of the redundant relation between genotype and phenotype. Genotypes are represented as squares. Two genotypes (sequences) folding into the same secondary structure belong to the same neutral network. Changes in a single nucleotide can lead to a complete rearrangement of the folded state, and thus to a significantly different phenotype. Typically, the genome space of RNA folding is such that many different phenotypes can be attained by changing only a few positions in the sequence.

not cause any visible change. However, if a better phenotype is encountered through this silent evolution behind the curtain, it will be fixed in the population rapidly (due to its advantage compared to the previously dominating one) in what will be interpreted as a punctuation of the dynamics. Note, however, that the population will then be genomically trapped in a position of the neutral network close to the old phenotype. It will take a while until it diffuses again on the new network and is able to access different, maybe improved, phenotypes.

Punctuated equilibrium was first defined in relation to the fossil record (Eldredge and Gould, 1972), and yet we have used a simple computational model for RNA folding to describe it. The question arises: has this process been observed also at the molecular level in natural systems? And the answer is yes. The process of spreading on a neutral network followed by a selective sweep when the population discovers a new, fitter phenotype, plus the subsequent exploration (again spreading) without phenotypic change to repeat the discovery of innovation, and so on, has been observed in the yearly dynamics of influenza A (Koelle et al., 2006). This dynamics describes the replacement every 2 to 8 years of circulating populations (where all individuals share a genetically similar hemaglutinine), by new populations, different from the previous one (but whose individuals again share similar sequences). Hemaglutinine is a protein that determines the antigenic properties of the virus: continuous changes in this protein permit influenza to escape immunity. This case constitutes a wonderful example of how relevant it is to use an appropriate genotype-phenotype map to understand the co-evolution of pathogens and hosts -or the adaptation properties of quasispecies.

\section{Fitness landscapes and eVOLUtion on NeUtral NETWORKS}

In order to understand the complex interplay between the fitness of genomes (which is determined by the adaptation 
that they provide to a specific environment) and the topology of the genome space, different paradigmatic fitness landscapes have been devised. Their introduction has been very much conditioned by the interest in obtaining analytical results describing the dynamics of quasispecies and other complex populations, as well as the characteristics of the process of adaptation and of the mutation-selection equilibrium. One of the most popular fitness landscapes is the single-peak landscape. Usually, it is assumed that a privileged genotype has the largest fitness and all the rest have lower fitness, well below that of the fittest sequence, or even zero. The Fujiyama landscape is smoother (also more complex) since it assumes that fitness of genotypes decreases with the number of mutations with respect to the fittest type. At the other extreme, we find rugged landscapes, among which two prototypical examples are the random landscape, where each genotype is assigned a randomly and independently chosen fitness value, or Kauffman's NK-landscapes, in which each of the $N$ genes of a sequence contributes additively to the fitness of the genome, but its fitness value results from its epistatic interactions (typically random) with $K$ other genes. There is not much in between, where one would guess that realistic landscapes should lie.

But, according to the picture we have just drawn, fitness landscapes should incorporate the high redundancy observed in biological sequences. Now we know that genotypes organize themselves into regions of common phenotypes, which therefore have constant fitness and which spread all over the genome space, forming so-called neutral networks. We can then try to figure out what the prototypical fitness landscapes should look like when these neutral networks of common phenotypes are taken into account. This is what Fig. 7 summarizes. The top row of that figure sketches a representation of the single peak, the Fujiyama, and the random landscapes, as referred to single genotypes. The single peak exhibits a single point of high fitness in a sea of points of lower or zero fitness. In the Fujiyama landscape, points decrease in fitness as they get away from the optimum sequence. In the random landscape points have random fitness, independently of each other. The lower row of Fig. 7 shows the phenotype counterparts of these three archetypes. Points are arranged into networks of constant fitness (equal phenotype), so the single peak now shows one of this networks with high fitness surrounded by other networks of low fitness and by non-viable genotypes (zero fitness). The Fujijama landscape is now defined in terms of distance between phenotypes, producing a landscape not quite distinguishable from what a random landscape now looks like.

In order to describe evolution in these new fitness landscapes we need new mathematical tools to deal with neutral networks (Reidys and Stadler, 2002). Neutral networks can be described through a connectivity matrix $C$, whose elements are $c_{i j}=1$ if genotypes $i$ and $j$ are mutually accessible and 0 otherwise. Evolution and adaptation, understood as a process of search and fixation of fitter phenotypes, is conditioned by the topology of these connectivity matrices and by the relationships between them, understood as objects defined in the space of genomes. There are a number of results that relate the topology of those graphs with the equilibrium states of populations and the dynamics of adaptation on the neutral network. It has been shown that the distribution of a population evolving (i.e. replicating and mutating) on a neutral network is solely determined by the topological properties of $C$, and given by its principal eigenvector. In that configuration the population has evolved mutational robustness, since it is located in a region of the neutral network where the connectivity is as large as possible (thus where mutations affect as less as possible the current phenotype) (van Nimwegen et al., 1999). This maximal connectivity equals the spectral radius of $C$. Equilibrium properties are thus well described once $C$ is known.

The dynamics of adaptation on neutral networks are more difficult to fully quantify because, in principle, all eigenvalues of the matrix intervene in the transient towards equilibrium. In addition, the time required to reach the equilibrium configuration depends on the initial condition: it might differ in orders of magnitude (in units of generations) if the population enters the network through a particular node -as in the case of influenza A- or if all genomes are equally represented -as in in vitro experiments that begin with a large population of random sequences. It has been shown that time to equilibrium is inversely proportional to the mutation rate, such that homogeneous populations (low mutation rates) will have it difficult to develop high mutational robustness. In very general conditions, the dominant term in the time to equilibrium is proportional to the ratio between the second largest and the largest eigenvalue of $C$ (Aguirre et al., 2009). C- 

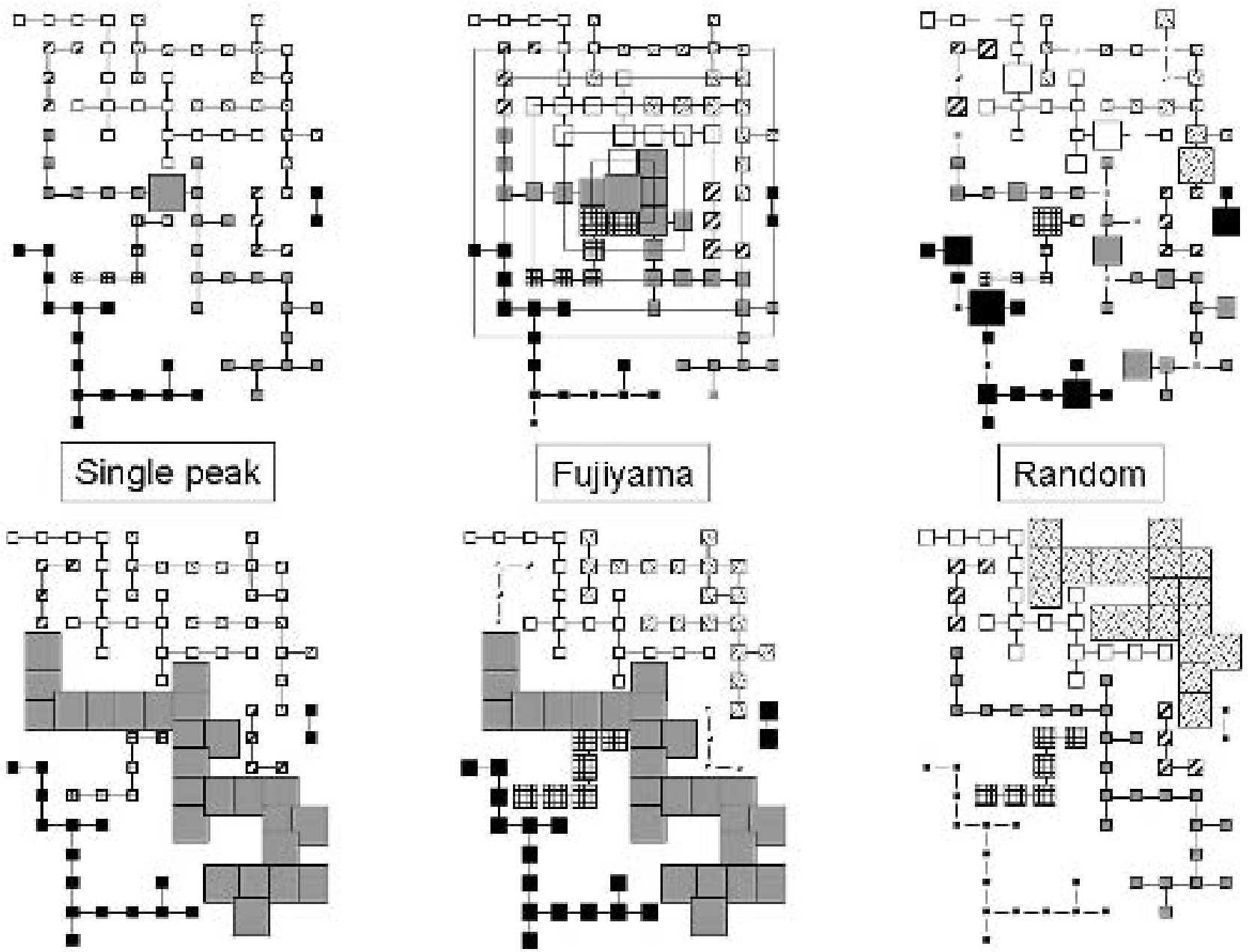

Figura 7. Schematic representation of three different fitness landscapes (as indicated) describing the differences between a genotype-based fitness and a phenotype-based fitness. Fitness values are proportional to the size of the nodes, and absent nodes are assumed to be non-viable sequences (zero fitness). The single peak landscape privileges one particular genome (above) or one particular phenotype (below). The Fujiyama landscape assigns maximum fitness to one sequence (above) or to one phenotype (below). Fitness decreases as the distance from each sequence or from each phenotype to the optimum increases. Note that this rule yields a smooth landscape only in sequence space, since phenotypes change much more abruptly. In the latter case, it resembles a random landscape (last column, below). The landscape defined by RNA folding shares many properties with random landscapes. A random assignation of fitness in genome space (last column, above) leads to a truly decorrelated landscape..

matrices are highly sparse, symmetric matrices for which it seems likely to develop approximations that could yield their two largest eigenvalues as a function of the average connectivity, for instance. To this end, the analysis of neutral networks could be performed in the limit of infinite size, given their exponentially fast growth in size with the sequence length.
Finally, an essential ingredient in the evolutionary process is randomness, and not only in relation to genetic drift. Random fluctuations play a main role in the searching process. Too low a variability in a population might even completely block adaptation. For example, the quantity that determines whether a population will be able to attain the region of maximal neutrality in finite time is the 
product of the population size times the mutation rate (van Nimwegen et al., 1999). Higher adaptability can be reached by means of a large population or through a large mutation rate. Overly small or homogeneous populations might get trapped in suboptimal configurations analogous to the metastable states observed in disordered systems.

A deeper knowledge of the topological properties of neutral networks and their mutual relationship in sequence space should lead to more realistic dynamical models for the evolution of populations. Provided one could characterize the fitness landscape, the probability of changing from one phenotype to another would be described through a matrix of transitions $M=\left(m_{i j}\right)$ between states, with $m_{i j} \geq 0$. This is actually a common formal framework to study population dynamics (Blythe and McKane, 2007). Matrices $M$ are stochastic, i.e. $\Sigma_{j} m_{i j}=1$ and thus define a homogeneous Markov chain. A full knowledge of the dynamics of the system amounts to knowing the eigenvalue spectrum of $M$.

\section{Conclusions}

The process of adaptation is not strongly relying on happy coincidences. The existence of huge and extensive neutral networks permits systematic explorations of the space of possible functions without paying a high fitness costs - a practical way to find out viable pieces later assembled to form complex individuals. Our current understanding of the relationship between genotype and phenotype clearly hints at the fact that even an evolutionary process restricted in the amount of change it can produce at the genomic level is not necessarily restricted in the amount of change it can cause at the phenotypic level. Further, it seems plausible that all possible phenotypes are sufficiently close to each other, such that it is not necessary to explore all the space of genotypes to find the optimal phenotype. While a genotype might be the needle in a haystack, you cannot help but stumble upon the phenotype.

This picture of a space of genomes where neutral networks corresponding to common functions are vastly extended and deeply interwoven has important implications in the way we understand and model the evolutionary process. Fast mutating populations, as RNA viruses, are able to spread rapidly and find new adaptive solutions thanks to the sustained generation of new viral types and the costless drift through large regions of genome space. Due to their relatively short genomes and the continuous accumulation of new mutations, it is very difficult (impossible in many cases) to trace the ancestry of extant viruses. Thus, viral phylogeny is located in evolutionary time, and the signal that speaks for its origins becomes increasingly weaker as we move backwards, until it is eventually lost. As a result, there is an on-going controversy on the origin of viruses, on their being a product of the postcellular era or the remnants of an ancient, pre-cellular RNA world. High mutation rates have been a successful strategy in their case, allowing the perpetual exploration of new genomic regions and thus escaping the attack of their hosts' defenses.

But when we come to talk about Life on Earth, with all the amazing complexity and diversity of organisms formed at least by one cell, it turns out that their common origins can be unequivocally identified. The phylogeny reconstructed through ribosomal units, single genes or whole genomes of living organisms clearly reveals the existence of LUCA, our Last Universal Common Ancestor, some 3.5 billion years ago. Is thus life on Earth resting on a frozen accident, that is the precise genomic pieces that formed LUCA? In the light of the above, we should answer "no". The first genomes could have occupied far-away places in the space of genomes and, still, it is highly improbable that functional life would look nowadays very different from the solutions (the phenotypes) we see all around us. 


\section{ACKNOWLEDGMENTS}

The authors acknowledge support from the Spanish Ministerio de Educación y Ciencia under projects FIS2008-05273 and MOSAICO, and from DGUI of the Comunidad de Madrid under the $R$ \& $D$ program of activities MODELICO-CM/S2009ESP-1691.

\section{NOTA}

1 Jean-Baptiste Lamarck's work, appeared in 1802, is considered the first -though incorrect- published theory of evolution. Lamarck's ideas were anticipated by Erasmus Darwin, one of Darwin's grand-fathers, and even earlier by Maupertuis, who envisioned a genetic inheritance of characters, entertained the idea that new species arise as mutant individuals, and even considered the elimination of deficient mutants, thus suggesting some kind of natural selection (Mayr, 1985).

\section{REFERENCES}

Aguirre, J., Buldú, J. M., and Manrubia, S. C. (2009): "Evolutionary dynamics on networks of selectively neutral genotypes: Effects of topology and sequence stability", Phys. Rev. E, 80:066112.

Ancel, L. W. and Fontana, W. (2000): "Plasticity, evolvability and modularity in RNA", J. Exp. Zool. (Mol. Dev. Evol.), 288: 242.

Anderson, P. C. and Mecozzi, S. (2005): "Unusually short RNA sequences: Design of 13-mer. RNA that selectively binds and recognizes theophylline", J.

Recibido: 10 de febrero de 2010 Aceptado: 15 de marzo de 2010 to evolutionary biology", J. Theor. Biol., 259:317-324.

Blythe, R. A. and McKane, A. J. (2007): "Stochastic models of evolution in genetics, ecology and linguistics", J. Stat. Mech., july: P07018.

Darwin, C. R. (1859): On the Origin of Species by Means of Natural Selection, or the Preservation of Favoured Races in the Struggle for Life, John Murray, London, 1st ed.

Domingo, E., Sabo, D., Taniguchi, T., and Weissmann, C. (1978): "Nucleotidesequence heterogeneity of an RNAphage population", Cell, 13: 735.

Duarte, E. A.; Novella, I. S.; Ledesma, S.; Clarke, D. K.; Moya, A.; Elena, S. F.; Domingo, E. and Holland, J. J. (1994): "Subclonal components of consensus fitness in an RNA virus clone", J. Virology, 6: 4295.

Eigen, M. (1971): "Selforganization of matter and evolution of biological macromolecules", Naturwissenschaften, 58: 465.

Eldredge, N. and Gould, S. J. (1972): "Punctuated equilibria: an alternative to phyletic gradualism", in Schopf, T. J. M., editor, Models in Paleobiology, pages 82-115. Freeman Cooper, San Francisco.

Ewens, W. J. (2004): Mathematical Population Genetic. I. Theoretical introduction, Springer, 2nd edition.

Fontana, W. (2002): "Modelling 'evo-devo' with RNA", BioEssays, 24: 1164.

Fontana, W. and Schuster, P. (1998): "Continuity in evolution: On the nature of transitions", Science, 280: 14511455.

Galton, F. (1886): "Regression towards mediocrity in hereditary stature", J. Anthrop. Inst., 15: 246-263.

Gould, S. J. (2002): The structure of evolutionary theory, Belknap Press of Harvard University Press. 
Grüner, W.; Giegerich, R.; Strothmann, D.; Reidys, C.; Weber, J.; Hofacker, I. L.; Stadler, P. F. and Schuster, P. (1996): "Analysis of RNA sequence structure maps by exhaustive enumeration. II. Structures of neutral networks and shape space covering", Monatshefte Chem., 127: 375-389.

Huxley, J. S. (1942): Evolution: The Modern Synthesis, Allen and Unwin.

Kimura, M. (1968): "Evolutionary rate at the molecular level", Nature, 217.

Koelle, K.; Cobey, S.; Grenfell, B. and Pascual, M. (2006): "Epochal evolution shapes the phylodynamics of interpandemic influenza A (H3N2) in humans", Science, 314: 1898-1903.

Mayr, E. (1985): The growth of biological thought, Belknap Press of Harvard University Press.
Mendel, G. (1866): "Versuche über Pflanzen-Hybriden", Verh. Naturforsh. Ver. Brünn, 4: 3-47.

Muller, H. J. (1932): "Some genetic aspects of sex", Amer. Nat., 66: 118-138.

Reidys, C. M. and Stadler, P. F. (2002): "Combinatorial landscapes", SIAM Rev., 44: 3-54.

Schultes, E. A. and Bartel, D. P. (2000): "One sequence, two ribozymes: Implications for the emergence of new ribozyme folds", Science, 289: 448452.

Schuster, P. (2006): "Prediction of RNA secondary structures: from theory to models and real molecules", Rep. Prog. Phys., 69: 1419.

Schuster, P.; Fontana, W.; Stadler, P. F. and Hofacker, I. L. (1994): "From sequences to shapes and back: A case study in RNA secondary structures", Proc. Roy. Soc. London B, 255: 279.

Sella, G. and Hirsh, A. E. (2005): "The application of statistical physics to evolutionary biology", Proc. Nat. Acad. Sci. USA, 102: 9541-9546.

Stich, M., Briones, C., and Manrubia, S. C. (2008): "On the structural repertoire of pools of short, random RNA sequences", J. Theor. Biol., 252: 750763.

van Nimwegen, E.; Crutchfield, J. P. and Huynen, M. (1999): "Neutral evolution of mutational robustness", Proc. Natl. Acad. Sci. USA, 96: 97169720.

Waterman, M. S. and Smith, T. F. (1978): "RNA secondary structure - Complete mathematical analysis", Math. Biosci., 42: 257. 